\title{
Reducing DDOS Attack Techniques in Cloud Computing Network Technology \\ T.Nathiya
}

Ph.D. Research Scholar Department of Computer Science, School of Computing Science, Vels University, Chennai - 639117, Tamil Nadu, India;

Abstract:

In cloud computing technology is most wanted in Information Technology and business organization. Network security is most important role in this technology. The security is one of the major issues, which reduce the growth of cloud computing resources and compliances to detect and preventing data's. The DDOS attack is attacking network resources in cloud. In this situation, many customers to avoid using cloud computing resources. To detect and prevent the Distributed Denial of Service(DDOS) attack in cloud. The cloud potentially to overcome the DDOS attack. The DDOS attack, DDOS attack architecture and DDOS attack various technologies, mitigation of DDOS, countermeasures of DDOS attack, how to process hardware checking methods and to detect and preventing DDOS attacks in tools.

Keywords- DDOS attack, techniques of DDOS, mitigation, network Security, Cloud Security

\section{Introduction}

Distributed computing is web based figuring condition where you pay just assets getting to. The client can utilize the cloud assets and administrations, for example, equipment and programming. Cloud giving conveyance model, for example, Infrastructure as an administration (Iaas), Platform as an administration (Paas) and Software as an administration (Saas). On-request arrange access to a mutual pool of figuring assets like system, server, stockpiling, applications and administration. Distributed computing give arrangement models, for example, private cloud, open cloud, half and half cloud and group cloud.

The fundamental information of cloud that is associated with PC to figuring assets to putting away information's and working with remote server can get to some related applications. Since the distributed computing condition utilizing a multi tenure and dispersed design actualizing cloud security alongside cloud conveyance show. The significant security issues of distributed computing are trustworthiness, security administration and information insurance.

The security cloud is giving by verification and get to control utilizing advanced character. In real security issues, DDOS (Distributed Denial of Service) assault. This assault is contaminated in system IP address into affected many cloud application and administrations. Its objective into expansive number of movement made in real user[1].

In Section 2, we talk about DDOS Attack, Section 3, we examine DDOS design and Section 4, we talk about DDOS strategies utilized as a part of distributed computing condition and Section 4, we examine aversion of DDOS assault lastly we talk about the conclusion.

\section{Proposed Work}

A standout amongst the best DDOS assaulted activity separately than genuine traffics. Turn around checking component is edge switches can be utilized to recognize the 
wellspring of parcels information. In the event that the DDOS assault a numerous datum will originating from casualty framework. In the event that the source IP address has overlooked in the sort of information will recognized. Utilizing TTL (Transistor transistor rationale) information parcel can be assembled. To play out this operation Hardware based checking innovation, equipment checking is keeping up in a table in the event that it will activity happen and have framework will be blocked. After certain time equipment checking, will check approaching parcels of information. This gadget safeguard machine to secure the DDOS assault particularly TCP SYN assault. Equipment checking and sifting innovation has proficient system against DDOS assault for any association to expend less assets. The proposed equipment based watermarking innovation to distinguish and keep the DDOS assault are taking after standards:

- The wellspring of the parcel recognized, when the bundle will achieve the system.

- Using Hop Count and TTL while instrument might be utilized to check the credibility of source address.

- If the source can't be checked that the bundle will dropped to the inward system.

- If the source is confirmed the parcels of information and association component checked to next confirmation step.

- Based on assault sort wellspring of parcel can be checked and drop the bundle and edge of system.

- Only trusted bundle can be set apart on the inner system.

\section{DDOS Attack}

Denial of Service(DOS) attack is a cyberthreat which is entered website or browser using online resources by client (such as pc, mobile phones, tablets). The DOS attack, in a criminal using a single network connection to software vulnerability or flood are targeted with fake request, these attempts are usually running into server resources like RAM, CPU, PROCESSER. The one is Distributed Denial of Service(DDOS) assaults are compose from various associating framework that are appropriated over the network[2]. DDOS assailant are utilize diverse IP locations to send distinctive sorts of information to the server or system. These procedures are extremely entangled for target server or system to separate between the viable activity and fake movement. The assailant's utilization ridiculed IP delivers as a source to send the information these circumstances are extremely convoluted. The DDOS assault might be utilize noteworthy business misfortune on the grounds that less profitability and administrations, increment downtime; thus, misfortune in notoriety. There are many motivations to utilizing DDOS assault in system asset to server. The primary reason many apparatuses accessible in DDOS attack[3]. A portion of the apparatuses might be utilized by aggressor yet exclude specialized aptitude. The greater part of them assailants get to the vast number of web utilizing into various $\mathrm{PC}$, which is utilized by DDOS assault. At last, the objective association to invest some energy to find the aggressor from asset, which help of Information Technology specialists. Numerous association doesn't to give the assets since some client urge the aggressor to direct the assault. It's high danger of losing association notoriety. The aggressor utilizing DDOS assault procedure a large portion of them association come to uncover security in broad daylight.

\section{Architecture of DDOS attack}

In this paper, Distributed denial of service attack is very hard to detect and mitigation, but 
few types of DDOS attack and some measure may to prevent and mitigation of them.

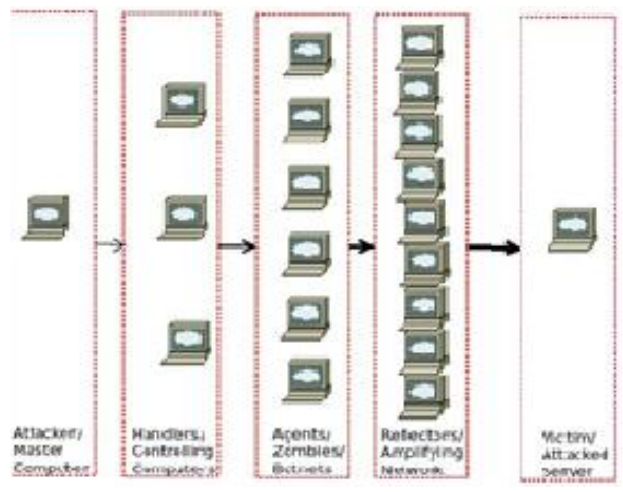

Figure 1: DDOS Attack Architecture

Above mention the figure 1 DDOS attack architecture diagram, which is separated into five components. There are two components main role - one is Attacker/ master computer to initiates the attack and another one is victim/Attacked server which is comes to attack by master computer. These two components are created denial of service attack. The three components in the middle one is Agent/Zombies/Botnet is to create a Denial of services of attacks are carried out.
The volunteers of computer or infected computer using internet browsing by the user, the user download malicious software, which is controlled by the attacker[4].

There additional layer of Handler/controlling computer which issuing instruction to the Agents and Botnet. And final components are reflecting layer which amplifies number of request to the victim/Attacked server. It's difficult to trace out the actual attacker.More over there is no IP address and IP address series of computer to connect the internet broadband connection, that computer to detect and blocked. Some attacker using computer don't directly connect to the victim server. The spoof IP address of victim server and send request to the large send to the victim server. But server confused which one is original reply.

\subsection{DDOS Attack Techniques}

There are more available DDOS Attack in cloud computing. We are discussing DDOS attack techniques methods.

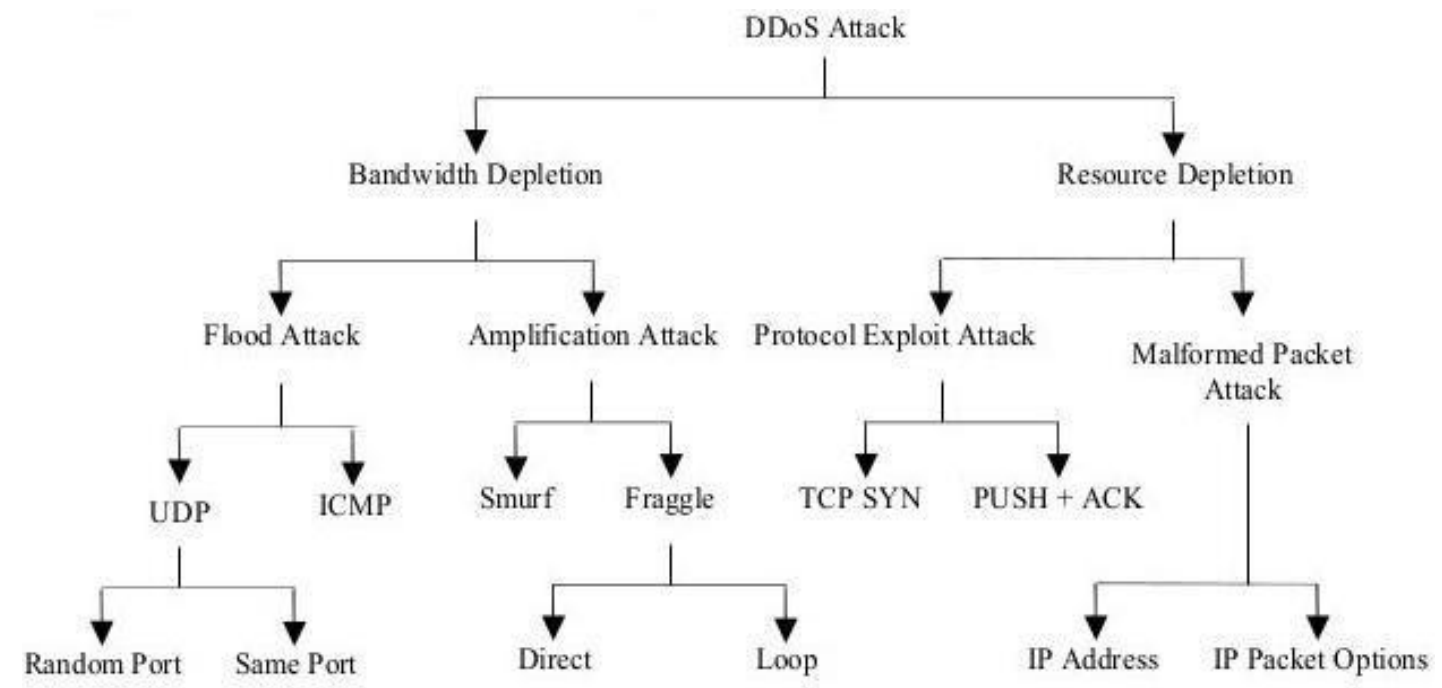

Figure 2: DDOS Attack Techniques 
In figure 2 DDOS assault strategies there are two primary classes, one is Bandwidth consumption and another is Resource exhaustion assaults. A data transfer capacity assault is an assault is intended to surge the system with accessible more movement in this way, to keep the honest to goodness activity from under the casualty framework. An asset exhaustion is intended to interface the asset of casualty framework to making procedure is appropriate demand for administration.

\subsection{Bandwidth Depletion Attacks}

Bandwidth attack is an attack which is classified into two class, one is flood attack and second one is Amplification attack.

\subsubsection{Flood Attack}

A surge assault is an assault that is included to make a lot of activity to a casualty frameworks. Organize data transfer capacity congested with IP movement to casualty framework. That why the casualty framework process is to back off, accidents from system transmission capacity to anticipating access by client. Surge assault is contaminated both UDP (User Datagram Protocol) and ICMP (Internet Control Message Protocol) bundles. In a UDP Flood assault is numerous parcels are send to recognized port on the casualty framework. In this framework attempt to get which application to send asking for data.The parody source IP address assaulting bundles utilizing assaulting DDOS devices. To conceal optional framework gives back the bundles from casualty framework. Be that as it may, casualty framework is not sent back to the Agent/Zombies3. An ICMP surge assault is process when the Agent sent some (ICMP_ECHO_REPLY) bundles to casualty framework. These casualty frameworks to answer the parcel flag and to consolidate activity data transfer capacity organize association of the casualty framework. Amid this ICMP surge assault is an ICMP parcel may likewise mock in the wellspring of IP address.

\subsubsection{Amplification Attack}

An Amplification Attack is a Bandwidth depletion based on Distributed denial of service (DDOS) attack. The attacker spoofed IP address request to Domain name server (DNS) to hide large number of source to direct response to the target server. These amplification is increase the size from 40 bytes to 4000 bytes it's nearly around Ethernet packet size. These quires broken down for transmission and then reassembled target network resources. A Botnet (it's a private network is infected some malicious software without owner knowledge) many amplified request an attacker to attacking bandwidth network resource. The attack is hard to protect against to come original request to server with valid traffic[5].

This Amplification attack is classified into two attack one is smurf attack and another one is fraggle attack. A smurf is a DDOS attack that spoofs ICMP_ECHO_REQ message to victim system. This process is slow down a network because thousands of packets to sending and network completely shutting down. A smurf attack is easy to infect the secure network system[6].

Another Attack is Fraggle attack is like smurf attack, the attacker to send the packet to network amplifier. In this DDOS attack that sending many spoofed UDP traffic to a router's broadcast network. The UDP fraggle packets character reached to target system. These frameworks each character to send the objective 
casualty framework. It will send reverberate parcel back to character generator and the procedure is rehashed. This produce awful movement harm the parcels than the smurf assault. This fraggle assault is characterized into two assault, Direct and Loop attacks[7].

\subsection{Resource Depletion}

DDOS assault asset exhaustion includes the aggressor sending the bundles to abuse arrange convention misuse interchanges and malware assets convention assault. The system suppliers tied up that why the left of honest to goodness client.

\subsubsection{Protocol Exploit Attacks.}

TCP SYN Attacks. The Transfer Control Protocol (TCP) to send the bundles of information amongst sender and collector. The framework sends a Synchronize (SYN) ask for and the accepting framework sends an Acknowledgment (ACK) to the SYN framework. The processor and memory assets held at the accepting framework for TCP SYN ask for until a timeout of transmission. In DDOS TCP SYN assault teaches the specialist to send TCP SYN ask for to the casualty server. In the server procedure stays dynamic and resend to the $\mathrm{ACK}+\mathrm{SYN}$ in parodied IP address. The server starts to come up short on processor and memory administrations. Assume the numerous TCP SYN assault demand is extensive and they are procedure to after some time the casualty framework will come up short on asset to react to any genuine user[8].

PUSH + ACK attacks. In the TCP convention to send the parcels of information to goal, the cushioned goal of TCP stack is full, the bundles to send on the getting framework. TCP stores approaching information in many pieces for message on to the accepting framework to limit the preparing framework. On the off chance that this procedure is rehashed with numerous casualty framework, the getting framework can't be process numerous approaching bundles information to the casualty framework will smashed.

\subsubsection{Malformed Packet Attack}

A distorted parcel assault is an assault, where the aggressor contaminates the operator to send mistakenly send the IP deliver of bundles to the casualty framework to be crash. There are two sorts of deformed parcel assault. One is IP address assault, in this assault contain same number of source and goal of IP locations. The Second IP bundle assault, to randomized IP parcel to preparing to casualty framework. The casualty framework to dissect the movement since they require extra preparing time. In the event that this assault is duplicated operator was utilizing so it can close down of the preparing casualty framework.

\section{DDOS Mitigation attack}

\subsection{Collaboration Method}

In this method to connecting many nodes to mitigates the attack and the victim server to get defected. Multiple nodes connected to mitigate the DDOS attack and victim server to get defected. Their main three stages to mitigate the DDOS attack such as firewall, pushback, and black holing.

\subsection{Non-Collaboration Method}


In this method involve many nodes but there is no collaboration between these nodes. During these nodes, no interaction for services, security applications and network resources. These method is classified into static and dynamic. The static model is not adapted to the attack. Dynamic work like an adaptive model, DDOS attack defense architecture will be automatically adjusted by mitigation method. The dynamic method is classified into redirecting and reconfiguration. The re-configuration is classified into three methods such as service, network and defense [9][10].

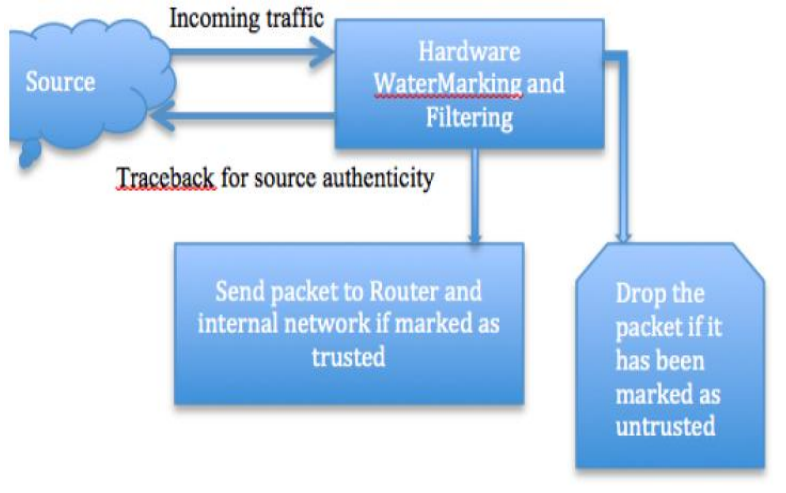

Figure 3: Hardware Based Watermarking Technology.

Hardware Based Watermarking Technology Verification Steps

Step 1: Maintain IP Address database History.

Step 2: Store IP Address.

Step 3: Store every moment movement circumstance and compute the normal of activity.

Step 4: If movement of system higher than consistent activity

Vol1 (1), July 2017, www.ijirase.com
At that point check IP Address. On the off chance that it's in database $\Rightarrow$ Access allowed

Else check IP Address more than 5 times in history database

On the off chance that yes then send it to IDS

Firewall proposed framework

Else checked IP and MAC address

On the off chance that checked $=>$ Access conceded

Else send it to IDS Firewall proposed framework.

\section{7. 7.Conclusion}

DDOS attack is using an attacking internal network IP address for legitimate user. If the critical system is affected in loss of economic network resources, loss of processing time in working times, loss of communication between network user. In this critical situation to detect and prevent the DDOS attacks. In cloud computing technologies DDOS attack infected in various ways. So, in this paper fully discuss in DDOS attack models and DDOS attacking different techniques, how to detect and prevent the DDOS attacks using watermarking technology in hardware based technology. The hardware based checking to prevent the network IP address from DDOS attack. In proposed paper, we will discuss in DDOS attack tools intrudes in network resource in Cloud computing environment. 
International Journal of Innovative Research in Applied Sciences and Engineering (IJIRASE) Volume 1, Issue 2, DOI: 10.29027/IJIRASE.v1.i1.2017.23-29, July 2017

\section{Reference}

1. Manjusha R, Ramachandran R. Secure authentication and access system for cloud computing auditing services using associated digital certificate. Indian Journal of Science and Technology. 2015 Apr 1; 8(7):1-8.

2. DDOS Protection center, Available from:https://www.incapsula.com/ddos/ddo s-attacks/denial-of-service.html.

3. Rajesh k. An Introduction to DDOSDistributed Denial of Service Attack, Network Security. Available form: http://www.excitingip.com/1500/anintroduction-to-ddos-distributed-denial-ofservice-attack/ , mar 2011.

4. Bijalwan Anchit and Singh Harvinder. Investigation of Bot Flooding Attack. Indian Journal of Science and Technology.2016 June Vol9(21).

5. Keyur Chauhan and Vivek Prasad." Distribution Denial of Service (DDOS) Attack Techniques and Prevention on Cloud Environment". International Journal of Innovation \& Advancement in Computer Science. Vol(4) sep 2015.

6. Michael J. Martin, "Smurf/fraggle attack defence using SACLs" Available form: http://searchnetworking.techtarget.com/ti p/Router-Expert-Smurf-fraggle-attackdefense-using-SACLs

7. DDOS Attack Definitions-DDOSPedia. Availableformhttps://security.radware.com /ddos-knowledgecenter/ddospedia/fraggleattack/

8. Nitesh Bharot et al." Mitigation Distributed Denial of Service Attack in Cloud Computing Environment using Threshold based Technique". Indian Journal of Science and Technology. Vol9 (38), Oct 2016.

9. Alireza S. Makan P, Fekih A. Taxonomy of Distributed Denial of service Mitigation Vol1 (1), July 2017, www.ijirase.com approaches for cloud computing. Elsevier Journal of Network and Computer Applications. 2015; 58:165-79.

10. Masudur Rahman and Wah man Cheung. A Novel Cloud Computing Security Model to Detect and Prevent DoS and DDoS Attack. International Journal of Advanced Computer Science and Applications. Vol 5, No 6, 2014. 